\title{
An Example of Non-Existence of Riley Equilibrium in Markets with Adverse Selection
}

\author{
Eduardo M. Azevedo* Daniel Gottlieb ${ }^{\dagger}$ \\ This version: March 25, 2019 \\ First version: January 21, 2016
}

\begin{abstract}
Rothschild and Stiglitz [1976] proposed a model of a competitive market with adverse selection and showed that a (pure strategy) Nash equilibrium may not exist. Among the solutions proposed to deal with this problem, a particularly influential one is the notion of Riley (or reactive) equilibrium [Riley, 1979]. We give an example that shows that a Riley equilibrium may not exist if consumers are not ordered.
\end{abstract}

Keywords. Adverse Selection, Equilibrium, Insurance.

*Wharton, eazevedo@wharton.upenn.edu.

${ }^{\dagger}$ Washington University in St. Louis, dgottlieb@wustl.edu. 
Rothschild and Stiglitz [1976] proposed a model of a competitive market with adverse selection where firms compete by offering insurance contracts to consumers. They showed that a (pure strategy) Nash equilibrium may not exist. Among the solutions proposed to deal with this problem, a particularly influential one is the notion of reactive equilibrium also known as Riley equilibrium [Riley, 1979]. In a Riley equilibrium, before introducing a new contract, each firm anticipates how other firms would react to it. If, in response to the new contract, another firm would introduce a profitable contract that makes the original firm lose money, it chooses not to introduce it. Additionally, this new profitable contract cannot be made unprofitable by the introduction of another contract.

Riley [1979] showed that the unique reactive equilibrium in the model of Rothschild and Stiglitz is the "least costly separating equilibrium." Engers and Fernandez [1987] generalized Riley's result, while maintaining the assumption of one-dimensional, ordered types. ${ }^{1}$ However, subsequent empirical and theoretical work has shown that consumers are often heterogeneous in more than one dimension, and that this is important for various policy questions. $^{2}$ In a recent influential paper, Handel et al. [2015] studied Riley equilibria in a setting that allows for richer heterogeneity. It is therefore natural to ask whether Riley equilibria exist more generally, allowing it to be used in empirically realistic settings.

In this note, we show that a Riley equilibrium may not exist. We give a natural example of a market with no Riley equilibria. As in Handel et al. [2015], the set of contracts is simple, as firms offer only two contracts. However, consumer types are not ordered along a single dimension, unlike the settings considered by Riley [1979], Engers and Fernandez [1987], and Handel et al. [2015]. This suggests that, to obtain predictions in more realistic environments, other solution concepts are necessary.

\section{Preliminaries}

We start with the formal definition of a Riley equilibrium based on Engers and Fernandez's [1987] generalization of Riley [1979]. ${ }^{3}$ The model studies the interaction between uninformed firms who sell different contracts to privately-informed consumers. Firms compete by offering

\footnotetext{
${ }^{1}$ As Rochet and Stole [2003] point out, there is a close relationship between models with multidimensional types and those with unordered types. For example, any model with finitely many multidimensional types can be written as a model with one-dimensional types, but the resulting one-dimension type space is typically not ordered.

${ }^{2}$ See, for example, Finkelstein and McGarry [2006], Cohen and Einav [2007], Fang et al. [2008]. Multidimensional heterogeneity is discussed in detail by Chiappori and Salanié [2000], Smart [2000], Heckman [2004], Araujo and Moreira [2010], Guerrieri and Shimer [2015], Veiga and Weyl [2016], Azevedo and Gottlieb [2017].

${ }^{3}$ See also Handel et al. [2015]. Our goal is to show that a Riley equilibrium may not exist. There is also a literature that studies whether static equilibrium concepts intended to capture dynamic adjustments, such as the Riley equilibrium, correspond to equilibria of a dynamic model (see, for example, Netzer and Scheuer [2014] and Mimra and Wambach [2016]).
} 
menus of contracts to consumers. Let $X=\left\{x_{1}, \ldots, x_{C}\right\}$ be the finite set of possible contracts. A price vector $\mathbf{p}=\left\{p_{1}, \ldots, p_{C}\right\}$ specifies a price for each contract.

Consider a situation where firms offer price vectors $\mathbf{p}^{1}, \ldots, \mathbf{p}^{N}$, and let $\mathbf{P}=\left\{\mathbf{p}^{1}, \ldots, \mathbf{p}^{N}\right\}$ be the set of price vectors being offered. In order to determine whether this is a Riley equilibrium, we must consider what happens when an entrant offers a new price vector $\mathbf{p}^{0}$. We write $\mathbf{p}^{0} \oplus \mathbf{P}$ to denote the new set of price vectors that is obtained by including vector $\mathbf{p}^{0}$ in $\mathbf{P}$, i.e., $\mathbf{p}^{0} \oplus \mathbf{P}=\left\{\mathbf{p}^{0}, \mathbf{p}^{1}, \ldots, \mathbf{p}^{N}\right\}$. For any $\mathbf{p} \in \mathbf{P}$, let $\Pi(\mathbf{p}, \mathbf{P})$ denote the per-unit profit obtained by a firm that offers price vector $\mathbf{p}$ when the set of all offered price vectors corresponds to $\mathbf{P}$. In applications, $\Pi$ is obtained by specifying preferences and the private information of informed consumers (as we will do in Section 2). A price vector $\mathbf{p} \in \mathbf{P}$ is safe if it cannot be made unprofitable by including any other vector $\mathbf{p}^{0}$. That is, $\Pi\left(\mathbf{p}, \mathbf{p}^{0} \oplus \mathbf{P}\right) \geq 0$ for any $\mathbf{p}^{0}$.

Definition 1. A Riley equilibrium is a set of price vectors $\mathbf{P}$ with the following properties:

- Firms do not incur losses: $\Pi(\mathbf{p}, \mathbf{P}) \geq 0$ for all $\mathbf{p} \in \mathbf{P}$.

- For any new price vector $\mathbf{p}^{A} \notin \mathbf{P}$ that gives a positive profit

$$
\Pi\left(\mathbf{p}^{A}, \mathbf{p}^{A} \oplus \mathbf{P}\right)>0
$$

there exists another price vector $\mathbf{p}^{B} \notin \mathbf{P}$ such that:

$\circ \mathbf{p}^{A}$ incurs losses when $\mathbf{p}^{B}$ is included:

$$
\Pi\left(\mathbf{p}^{A}, \mathbf{p}^{B} \oplus \mathbf{p}^{A} \oplus \mathbf{P}\right)<0,
$$

$\circ \mathbf{p}^{B}$ does not incur losses when any new price vector $\mathbf{p}^{C}$ (possibly $\emptyset$ ) is included $\left(\mathbf{p}^{B}\right.$ is "safe"). That is, for any $\mathbf{p}^{C}$,

$$
\Pi\left(\mathbf{p}^{B}, \mathbf{p}^{C} \oplus \mathbf{p}^{B} \oplus \mathbf{p}^{A} \oplus \mathbf{P}\right) \geq 0
$$

Definition 1 states that a set of price vectors $\mathbf{P}$ fails to be a Riley equilibrium if and only if either it makes negative profits, or there exists a profitable deviation $\mathbf{p}^{A}$ such that: either (a) $\mathbf{p}^{A}$ is safe, or (b) if $\mathbf{p}^{A}$ is not safe, any deviation $\mathbf{p}^{B}$ that makes it unprofitable must not be safe itself. In particular, taking $\mathbf{p}^{C}=\emptyset$, we find that $\mathbf{p}^{B}$ cannot incur losses when added to $\mathbf{p}^{A} \oplus \mathbf{P}$.

Riley equilibria generalize (pure strategy) Nash equilibria of a model of Bertrand competition by ruling out any deviation that could be made unprofitable by some other deviation, as long as this other deviation is safe. Therefore, when there are no safe prices, Riley equilibria 
and (pure strategy) Nash equilibria coincide. This observation will be useful in our proof of Lemma 2 below.

\section{An Example of Non-Existence of Riley Equilibria}

We now show that Riley equilibria may not exist when types are not ordered. Our example is based on a variation of the Hotelling model. Consumers are uniformly distributed in the unit interval $\theta \in[0,1]$. There are two possible contracts: $X=\{0,1\}$. A type- $\theta$ consumer who buys contract $x$ at price $p$ gets utility

$$
K-\frac{(x-\theta)^{2}}{\alpha}-p,
$$

where $\alpha>0$ parameterizes the similarity between contracts (it is the inverse of the transportation cost in the standard Hoteling model) and $K$ is large enough to ensure that the market is served. The model has adverse selection, in the sense that the cost of selling a contract depends on the consumer who is buying it. Selling contract $x \in\{0,1\}$ to type $\theta$ costs

$$
\left(\theta-\frac{1}{2}\right)^{2}
$$

The key aspect of our example is that types are not ordered along a single dimension in preferences and costs. The lack of ordering is a key driver of non-existence, because Riley [1979] and Handel et al. [2015] have obtained existence theorems under the assumption of ordered types. ${ }^{4}$ In our model, preferences are ordered in one dimension by $\theta$ in $[0,1]$, which measures how much a consumer likes contract $x=1$ relative to contract $x=0$. However, costs are not monotonic in $\theta$. The cheapest consumers are the intermediate ones, with $\theta=1 / 2$, whereas the most expensive are the consumers with extreme values of $\theta=0$ and $\theta=1$. Therefore, consumers cannot be ordered in a single dimension summarizing both their costs and preferences.

Before proving that a Riley equilibrium does not exist under certain parameters, we briefly discuss the intuition. This discussion is not necessary for the proofs and may be skipped. Figure 1 plots costs as a function of type $\theta$. To make the discussion more intuitive, suppose prices are measured in thousands of dollars. The most expensive types $\theta=0$ and $\theta=1$ have a cost of $1 / 4(\$ 250)$. Consider Nash equilibria first. A plausible candidate is for both contracts to have the same price. Consumers with $\theta$ in $[0,1 / 2]$ buy contract 0 , and consumers with $\theta$ in $[1 / 2,1]$ buy contract 1 . For firms to make zero profits, the price has to be equal

\footnotetext{
${ }^{4}$ For example, in Handel et al. [2015], there are two contracts, one with $90 \%$ coverage and another with $60 \%$ coverage. For each type, the cost of providing the $90 \%$ contract is greater than the cost of providing the $60 \%$ contract. Moreover, consumers with higher types have a higher preference for the $90 \%$ coverage and are are more expensive to cover.
} 


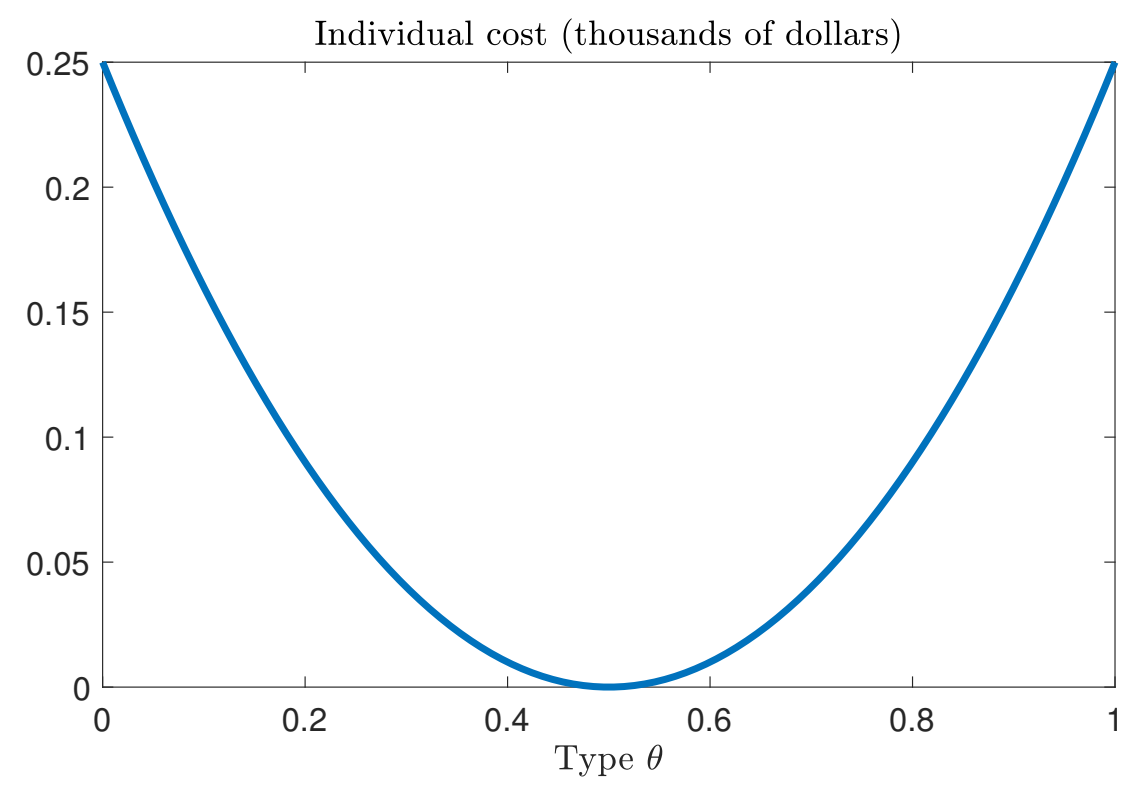

Figure 1: Unit cost as a function of consumer type.

to the average cost in Figure 1, which is 1/12 (\$83.33). However, Lemma 1 shows that this is not an equilibrium when the demand is very elastic (that is, $\alpha$ is high). A firm can profit by offering contract 0 for a slightly lower price, such as $\$ 80$. This corresponds to a deviation such as $\mathbf{p}^{A}=(\$ 80, \infty)$. The firm will gain all of the market share of contract 0 and will attract some marginal consumers close to $\theta=1 / 2$, who are the cheapest consumers. The upshot is that no Nash equilibrium exists when $\alpha$ is high (as demonstrated by Lemma 1).

The more interesting question is whether a Riley equilibrium exists. For example, it could be that pricing both contracts at $1 / 12$ (that is, \$83.33) is not a Nash equilibrium but is a Riley equilibrium. If so, then a deviation like $\mathbf{p}^{A}=(\$ 80, \infty)$ must lose money if another safe deviation $\mathbf{p}^{B}$ is added. For example, a competitor could try to offer $\mathbf{p}^{B}=(\infty, \$ 77)$, which would steal low-cost consumers from the firm offering $\mathbf{p}^{\mathbf{A}}$. The key point is that no such deviation $\mathbf{p}^{B}$ is safe. The reason is that there is always a deviation $\mathbf{p}^{C}$ that steals even more of the lower-cost consumers from the firm offering $\mathbf{p}^{B}$, making $\mathbf{p}^{B}$ unprofitable. Formally, Claim 1 shows that, for sufficiently high $\alpha$, it is only safe to make offers that are above the highest possible cost of $1 / 4$ (that is, $\$ 250$ ). Thus, the different ordering of preferences and costs makes it so that there are few safe deviations, so that a Riley equilibrium also does not exist. 


\section{Proof that there is no Equilibrium}

The type $\theta^{*}$ who is indifferent between both contracts when the lowest prices are $p_{0}$ and $p_{1}$ is determined by:

$$
K-\frac{\theta^{2}}{\alpha}-p_{0}=K-\frac{(1-\theta)^{2}}{\alpha}-p_{1} \therefore \theta^{*}=\frac{1-\alpha\left(p_{0}-p_{1}\right)}{2} .
$$

Then, because types are uniformly distributed, the demand for contract 0 is:

$$
D_{0}\left(p_{0}, p_{1}\right) \equiv\left\{\begin{array}{c}
0 \text { if } 1+\alpha\left(p_{1}-p_{0}\right)<0 \\
\frac{1+\alpha\left(p_{1}-p_{0}\right)}{2} \text { if } 0 \leq 1+\alpha\left(p_{1}-p_{0}\right) \leq 2 \\
1 \text { if } 1+\alpha\left(p_{1}-p_{0}\right)>2
\end{array} .\right.
$$

By symmetry, the demand for contract 1 is analogous. We say that a price vector $\left(p_{0}, p_{1}\right)$ yields an interior allocation if $0<D_{0}\left(p_{0}, p_{1}\right)<1$.

Because costs are symmetrically distributed, we can define $C(q)$ as the expected per-unit cost of selling to the mass $q$ of consumers with the highest willingness to pay for each contract:

$$
C(q)=\frac{\int_{0}^{q}\left(\theta-\frac{1}{2}\right)^{2}}{q} d \theta=\frac{q^{2}}{3}-\frac{q}{2}+\frac{1}{4} .
$$

For interior allocations, the per-unit profit of selling $x=i$ at price $p_{i}$ equals

$$
p_{i}-C\left(D_{i}\left(p_{i}, p_{-i}\right)\right)=p_{i}-\frac{1-\alpha\left(p_{-i}-p_{i}\right)+\alpha^{2}\left(p_{-i}-p_{i}\right)^{2}}{12} .
$$

The per-unit profit function $\Pi$ can be easily calculated from equation (2). ${ }^{5}$

It is helpful to consider the existence of Nash equilibria before turning to Riley equilibria. Lemma 1 shows that Nash equilibria do not exist when horizontal differentiation between contracts is low (i.e., $\alpha$ is high). ${ }^{6}$

\footnotetext{
${ }^{5}$ To do so, let $p_{i}^{*}(\mathbf{P}) \equiv \min \left\{\tilde{p}_{0}:\left(\tilde{p}_{0}, \tilde{p}_{1}\right) \in \mathbf{P}\right\}$ denote the lowest price being charged for contract $i \in\{0,1\}$. The per-unit profit of selling contract $i$ is

$$
\phi_{i}\left(p_{i}, \mathbf{P}\right) \equiv \begin{cases}0 & \text { if } p_{i} \neq p_{i}^{*}(\mathbf{P}) \text { or } p_{i}^{*}(\mathbf{P})-p_{-i}^{*}(\mathbf{P}) \geq \frac{1}{\alpha} \\ p_{i}-\frac{1-\alpha\left(p_{-i}-p_{i}\right)+\alpha^{2}\left(p_{-i}-p_{i}\right)^{2}}{12} & \text { if } p_{i}=p_{i}^{*}(\mathbf{P}) \text { and }\left|p_{i}^{*}(\mathbf{P})-p_{-i}^{*}(\mathbf{P})\right|<\frac{1}{\alpha} \\ p_{i}-\frac{1}{12} & \text { if } p_{i}=p_{i}^{*}(\mathbf{P}) \text { and } p_{-i}^{*}(\mathbf{P})-p_{i}^{*}(\mathbf{P}) \geq \frac{1}{\alpha}\end{cases}
$$

The first line says that the firm gets zero profits if some other firm is charging a lower price, or if no consumer is buying contract $i$. The second line specifies the per-unit profits if the allocation is interior, whereas the third line specifies profits when all consumers buy contract $i$. Total per-unit profits are the sum of per-unit profits from both contracts:

$$
\Pi\left(\left(p_{0}, p_{1}\right), \mathbf{P}\right) \equiv \phi_{0}\left(p_{0}, \mathbf{P}\right)+\phi_{1}\left(p_{1}, \mathbf{P}\right) .
$$
}

${ }^{6}$ This is a natural result in light of existence problems with price competition and consumers differentiated 
Lemma 1. Let $\alpha>12$. Then, no (pure strategy) Nash equilibrium exists.

Proof. In any (pure strategy) Nash equilibrium, profits on all contracts are zero. Let $\left(p_{0}, p_{1}\right)$ denote the lowest prices charged for each contract. With interior allocations, setting per-unit profits (2) equal to zero for both contracts yields $p_{0}=p_{1}$ as long as $\alpha \neq 6$. Thus, there are three possible Nash equilibria: an interior, symmetric Nash equilibrium $\left(p_{0}=p_{1}\right)$ and two asymmetric Nash equilibria at the boundaries (one where all consumers buy $x=0$ and another where they all buy $x=1$ ). In the symmetric candidate equilibrium, we must have

$$
p_{0}=p_{1}=C\left(\frac{1}{2}\right)=\frac{1}{12} .
$$

In the asymmetric candidate equilibria, zero profits gives:

$$
p_{i}=C(1)=\frac{1}{12}
$$

where $i \in\{0,1\}$ denotes the only contract being sold. Notice that, in all candidate equilibria, all traded contracts have price $\frac{1}{12}$.

We now verify that these are not Nash equilibria because firms can profit by offering a contract at a price slightly below $\frac{1}{12}$ and attracting a large mass of low-cost consumers. In the symmetric candidate equilibrium, the per-unit profit from selling a contract at price $p<\frac{1}{12}$ is

$$
p-\frac{1-\alpha\left(\frac{1}{12}-p\right)+\alpha^{2}\left(\frac{1}{12}-p\right)^{2}}{12} .
$$

The derivative of this expression with respect to $p$ equals

$$
1-\frac{\alpha-2 \alpha^{2}\left(\frac{1}{12}-p\right)}{12}
$$

which, evaluated at $p=\frac{1}{12}$, becomes

$$
1-\frac{\alpha}{12}<0 \Longleftrightarrow \alpha>12
$$

Therefore, a small reduction in price below $\frac{1}{12}$ yields positive profits whenever $\alpha>12$. Next, consider the asymmetric candidate equilibrium where only contract $x=i$ is sold at price $p_{i}=\frac{1}{12}$. By the same calculations as before, selling the other contract $(x \neq i)$ at a price slightly below $\frac{1}{12}$ yields positive profits. Thus, no (pure strategy) Nash equilibrium exists when $\alpha>12$.

a la Hotelling, even without adverse selection. In these models, much like in ours, profits can be discontinuous as a function of prices, and a firm's best response might not be convex-valued. See Caplin and Nalebuff [1991] pp. 26-27 and references therein for an explanation of the existence issues in this type of model. 
We now turn to the existence of Riley equilibria. Before doing so, it is helpful to introduce some terminology. We say that a price vector $\mathbf{p}$ is safe if there is no other price vector $\mathbf{p}^{A}$ that could make $\mathbf{p}$ unprofitable:

$$
\Pi\left(\mathbf{p},\left\{\mathbf{p}, \mathbf{p}^{A}\right\}\right)<0 .
$$

Our next result shows that the set of Riley equilibria coincides with the set of Nash equilibria when $\alpha>4$ :

Lemma 2. Let $\alpha>4$. Then, a set of price vectors $\mathbf{P}$ is a Riley equilibrium if and only if it is a (pure strategy) Nash equilibrium.

The proof will follow from two claims. First, we will show that a price vector is safe if and only if all prices are at least $\frac{1}{4}$. Because there are no safe prices below $\frac{1}{4}$, this result implies that, if we restrict to prices in this range, price vectors in Riley and Nash equilibria coincide. Second, we will show that there are neither Riley nor Nash equilibria in which all prices are above $\frac{1}{4}$.

Claim 1. A price vector $\left(p_{0}, p_{1}\right)$ is safe if and only if $p_{0} \geq \frac{1}{4}$ and $p_{1} \geq \frac{1}{4}$.

Proof. To see that any vector that charges at least $\frac{1}{4}$ for all contracts must be safe, notice that the cost of supplying a contract to type $\theta$ is $\left(\theta-\frac{1}{2}\right)^{2}$, which has a maximum value of $\frac{1}{4}$. Therefore, no contract with a price above $\frac{1}{4}$ can be unprofitable.

We now show that $\left(p_{0}, p_{1}\right)$ is not safe when either $p_{0}<\frac{1}{4}$ or $p_{1}<\frac{1}{4}$. Without loss of generality, let $p_{0} \leq p_{1}$ and suppose that $p_{0}<\frac{1}{4}$ (the case where $p_{1}<p_{0}$ is symmetric). Let $\mathbf{p}_{\epsilon}^{\mathbf{A}} \equiv\left(p_{0}+1, p_{0}-\frac{1}{\alpha}+\epsilon\right)$, where $0<\epsilon<p_{1}-p_{0}+\frac{1}{\alpha}$. Substituting in (1) verifies that a positive mass of consumers buys each of the two contracts (contract 0 at price $p_{0}$ and contract 1 at price $\left.p_{0}-\frac{1}{\alpha}+\epsilon\right)$. A firm offering $\mathbf{p}$ sells contract $x=0$ at price $p_{0}$, making a profit of

$$
p_{0}-\frac{1-\alpha\left(p_{1}^{A}-p_{0}\right)+\alpha^{2}\left(p_{1}^{A}-p_{0}\right)^{2}}{12}=p_{0}-\frac{2-\alpha \epsilon+\alpha^{2}\left(\frac{1}{\alpha}-\epsilon\right)^{2}}{12}
$$

which converges to $p_{0}-\frac{1}{4}$ as $\epsilon \searrow 0$. Since $p_{0}<\frac{1}{4}$, offering price vector $\mathbf{p}$ is unprofitable after $\mathbf{p}_{\epsilon}^{A}$ is introduced when $\epsilon$ small enough, showing that $\mathbf{p}$ is not safe.

Claim 2. Let $\alpha \neq 6$. There are no Riley equilibrium and no (pure strategy) Nash equilibrium in which all prices are weakly greater than $\frac{1}{4}$.

Proof. We first show that in any (pure strategy) Nash equilibrium of the Bertrand game, each traded contract makes zero profits. To see this, notice that if a firm makes strictly positive profits from a contract, a firm that undercuts the price of this contract by a small amount would capture the entire market for this contract at approximately the same per-unit profit (since per-unit profits 2 are continuous in prices), thereby obtaining strictly positive profits. 
Recall that, as shown in the proof of Lemma 1, any traded contract that makes zero profits must be sold at price $\frac{1}{12}<\frac{1}{4}$. Therefore, we cannot have a Nash equilibrium with any contract traded at prices weakly above $\frac{1}{4}$.

Next, we show that there are no Riley equilibria with prices strictly above $\frac{1}{4}$. Notice that we cannot have an interior Riley equilibrium in which at least one price is strictly above $\frac{1}{4}$ (otherwise, introducing the price vector $\mathbf{p}^{A}=\left(\frac{1}{4}, \frac{1}{4}\right)$ is both safe and profitable). There are two possibilities: (i) one contract is sold at price $\frac{1}{4}$ and the price of the other contract is high enough that no one buys, and (ii) both contracts are sold at price $\frac{1}{4}$.

To see that case (i) cannot be a Riley equilibrium, let $\mathbf{P}$ be a set of price vectors where $p_{0}=\frac{1}{4}$ is the lowest price offered for contract 0 and the lowest offered for contract 1 satisfies $p_{1}>\frac{1}{4}+\frac{1}{\alpha}$. Under these prices, all types buy contract 0 .

To verify that $\mathbf{P}$ is not a Riley equilibrium, it suffices to show that there exists a price vector $\mathbf{p}^{A}$ that makes a strictly positive profit when added to this market $\left(\Pi\left(\mathbf{p}^{A}, \mathbf{p}^{A} \oplus \mathbf{P}\right)>0\right)$ and that does not incur any losses when any other $\mathbf{p}^{B}$ is added $\left(\Pi\left(\mathbf{p}^{A}, \mathbf{p}^{B} \oplus \mathbf{p}^{A} \oplus \mathbf{P}\right) \geq 0\right.$ for all $\mathbf{p}^{B}$ ). But this follows directly from Claim 1 (adding $\mathbf{p}^{A}=\left(1, \frac{1}{4}\right)$ gives a per-unit profit of $\frac{1}{6}>0$ when included to $\mathbf{P}$, and $\mathbf{p}^{A}$ cannot be made unprofitable by introducing any price vector $\mathbf{p}^{B}$ to $\left.\mathbf{p}^{A} \oplus \mathbf{P}\right)$.

Next, we verify that case (ii) cannot be a Riley equilibrium. To show that a set of price vectors $\mathbf{P}$ is not a Riley equilibrium, it suffices to show that for any profitable deviation $\mathbf{p}^{A}$, any deviation $\mathbf{p}^{B}$ that makes $\mathbf{p}^{A}$ unprofitable is not safe.

Let $\mathbf{P}$ be a set of price vectors under which both contracts are sold at price $\frac{1}{4}$. Note that there exist price vectors $\mathbf{p}^{\mathbf{A}} \leq\left(\frac{1}{4}, \frac{1}{4}\right)$ that make strictly positive profits (since per-unit profits 2 are continuous in prices and the per-unit profit of offering $\left(\frac{1}{4}, \frac{1}{4}\right)$ is $\left.\frac{1}{6}>0\right)$. Note also that a new contract $\mathbf{p}^{\mathbf{A}}$ can only be profitable if it offers at least one contract (say, $x=0$ ) at a price $p_{0}^{A}<\frac{1}{4}$ (otherwise, no consumer would buy from $\mathbf{p}^{A}$ and it would make zero profits). In line with Definition 1 , let $\mathbf{p}^{B}$ be a new price vector that, when added to $\mathbf{p}^{A} \oplus \mathbf{P}$, makes $\mathbf{p}^{A}$ unprofitable. This can only be true if either $p_{0}^{B}<p_{0}^{A} \leq \frac{1}{4}$ (introducing $\mathbf{p}^{B}$ steals all customers who were buying $x=0$ from $\mathbf{p}^{A}$ ) or $p_{1}^{B}<\frac{1}{4}$ (introducing $\mathbf{p}^{B}$ affects the pool of customers who buy $x=0$ from $\mathbf{p}^{A}$, increasing the average cost of selling $x=0$ ). By Claim 1 , such $\mathbf{p}^{B}$ exists. Moreover, also by Claim $1, \mathbf{p}^{B}$ is not safe, meaning that there is always a vector $\mathbf{p}^{C}$ that, when included to $\mathbf{p}^{B} \oplus \mathbf{p}^{A} \oplus \mathbf{P}$, makes $\mathbf{p}^{B}$ itself unprofitable.

Combining Lemmas 1 and 2 gives our main result:

Proposition 1. No Riley equilibrium exists when $\alpha>12$.

While Riley equilibria may not exist, the model always has a competitive equilibrium, as defined by Azevedo and Gottlieb [2017]. The interior allocation in which both contracts are traded at price $\frac{1}{12}$ is always an equilibrium. In addition, when $\alpha \leq 6$ (so that horizontal differentiation between contracts is high enough), there are also two equilibria in which only 
one of the contracts is traded at price $\frac{1}{12}$ and the non-traded contract is supplied at price

$\frac{1}{12}+\frac{1}{\alpha}$. From Dasgupta and Maskin [1986], the model also has a Nash equilibrium in mixed strategies. Another possible solution is to rewrite the model in a dynamic setting, such as in Mimra and Wambach [2016], Netzer and Scheuer [2014].

\section{Conclusion}

Starting with Rothschild and Stiglitz [1976], a large theoretical literature has studied equilibria in adverse selection models with one-dimensional types. One of the most commonly used equilibrium concepts is that of Riley [1979]. In recent years, however, several studies have emphasized the importance of incorporating heterogeneity in more than one dimension. This paper shows that the Riley equilibrium may fail to exist if one drops the assumption of one-dimensional types.

\section{References}

Aloisio Araujo and Humberto Moreira. Adverse selection problems without the spencemirrlees condition. Journal of Economic Theory, 145(3):1113-1141, 2010.

Eduardo M Azevedo and Daniel Gottlieb. Perfect competition in markets with adverse selection. Econometrica, 85(1):67-105, 2017.

Andrew Caplin and Barry Nalebuff. Aggregation and imperfect competition: On the existence of equilibrium. Econometrica, pages 25-59, 1991.

Pierre-André Chiappori and Bernard Salanié. Testing for asymmetric information in insurance markets. Journal of Political Economy, 108(1):56-78, 2000.

Alma Cohen and Liran Einav. Estimating risk preferences from deductible choice. American Economic Review, 97(3):745-788, 2007.

Partha Dasgupta and Eric Maskin. The existence of equilibrium in discontinuous economic games, i: Theory. Review of Economic Studies, 1(53):1-26, 1986.

Maxim Engers and Luis Fernandez. Market equilibrium with hidden knowledge and selfselection. Econometrica, 55(2):425-439, 1987.

Hanming Fang, Michael P Keane, and Dan Silverman. Sources of advantageous selection: Evidence from the medigap insurance market. Journal of Political Economy, 116(2):303$350,2008$. 
Amy Finkelstein and Kathleen McGarry. Multiple dimensions of private information: Evidence from the long-term care insurance market. American Economic Review, 96(4): 938-958, 2006.

Veronica Guerrieri and Robert Shimer. Markets with multidimensional private information. Mimeo, University of Chicago, 2015.

Benjamin Handel, Igal Hendel, and Michael D Whinston. Equilibria in health exchanges: Adverse selection vs. reclassification risk. Econometrica, 83(4):1261-1313, 2015.

James J Heckman. Lessons from the technology of skill formation. Annals of the New York Academy of Sciences, 1038(1):179-200, 2004.

Wanda Mimra and Achim Wambach. A note on uniqueness in game-theoretic foundations of the reactive equilibrium. Economics Letters, 141:39-43, 2016.

Nick Netzer and Florian Scheuer. A game theoretic foundation of competitive equilibria with adverse selection. International Economic Review, 55(2):399-422, 2014.

John G Riley. Informational equilibrium. Econometrica, pages 331-359, 1979.

Jean-Charles Rochet and Lars A Stole. The economics of multidimensional screening. In Mathias Dewatripont, Lars Peter Hansen, and Stephan Turnovsky, editors, Advances in Economics and Econometrics: Theory and Applications. Cambridge University Press, Cambridge, 2003.

Michael Rothschild and Joseph Stiglitz. Equilibrium in competitive insurance markets: An essay on the economics of imperfect information. Quarterly Journal of Economics, 90(4): 629-649, 1976.

Michael Smart. Competitive insurance markets with two unobservables. International Economic Review, 41(1):152-169, 2000.

André Veiga and E Glen Weyl. Product design in selection markets. Quarterly Journal of Economics, 131(2):1007-1056, May 2016. 